\title{
Systematic Review: Assessment of the Possibility of Vertical Transmission of COVID-19: A Systematic Review and Meta-analysis Protocol
}

\author{
Sareh Dashti $^{1}$ (D), Tahereh Fathi Najafi ${ }^{1}$ (D, Hamid Reza Tohidinik² (D) $_{\text {Narjes Bahri }}{ }^{*}$ (D) \\ 1. Department of Midwifery, Mashhad Branch, Islamic Azad University, Mashhad, Iran \\ 2. HIV/STI Surveillance Research Center, and WHO Collaborating Center for HIV Surveillance, Institute for Futures Studies in Health, Kerman Uni- \\ versity of Medical Sciences, Kerman, Iran. \\ 3. Department of Midwifery, Social Development and Health Promotion Research Center, Faculty of Medicine, Gonabad University of Medical Sciences, \\ Gonabd, Iran
}

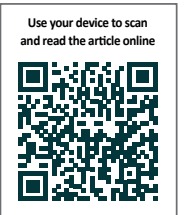

dtation Dashti S, Fathi Najafi T, Tohidinik HR, Bahri N. Assessment of the Possibility of Vertical Transmission of COVID-19: A Systematic Review and Meta-analysis Protocol. Journal of Research \& Health. 2021; 11(2):71-76. http://dx.doi. org/10.32598/JRH.11.2.72.3

\section{http://dx.doi.org/10.32598/JRH.11.2.72.3}

\section{Keywords:}

COVID-19, Coronavirus,

Vertical transmission, Infectious disease transmission

\section{ABSTRACT}

Background: The Coronavirus Disease 2019 (COVID-19) outbreak has put a great burden on global health and healthcare systems. There is controversy regarding the possibility of vertical transmission of COVID-19. This proposed systematic review will be done to assess the possibility of vertical transmission of COVID-19 based on currently published literature.

Methods: In this study, all published observational studies, including cross-sectional, cohort, and case-control studies, as well as case reports and case series, in peer-reviewed journals in any language until the end of July 2020 will be assessed. Editorials, commentaries, and letters to editors will be excluded from the review. Searching will be conducted using international bibliographic databases, including PubMed, Embase, and Web of Science based on Preferred Reporting Items for Systematic reviews and Meta-Analysis (PRISMA) checklist. The search strategy will be improved and finalized based on the results of the primary search. Data extraction of the included articles will be performed by two researchers using the Zotero and review manager (revMan) software. The heterogeneity of the articles will be assessed using DerSimonian \& Laird $\mathrm{Q}$ test and $\mathrm{I}^{2}$ statistic. The pooled estimated prevalence of vertical transmission of COVID-19 will be performed using the Metaprop command. Publication bias will be assessed using Begg's rank correlation and the Egger weighted regression methods

Results: Based on the reviewers comments the results section was deleted because this manuscript was a systematic review protocol.

Conclusion: The findings of this study will help practitioners and health care providers in decision-making for the care and management of COVID-19-infected pregnant women.

\footnotetext{
*Corresponding Author:
}

Narjes Bahri, PhD.

Address: Department of Midwifery, Social Development and Health Promotion Research Center, Faculty of Medicine, Gonabad University of Medical Sciences, Gonabd, Iran.

Phone: +98 (51) 57223028

E-mail:nargesbahri@yahoo.com 


\section{Introduction}

he Coronavirus disease 2019 (COVID-19) began circulating in Wuhan, $\mathrm{Hu}$ $\mathbf{T}$ bei province, China in December 2019 It is a highly contagious disease, and can wildly and rapidly spread through respiratory droplets of infected individuals [1]. The ongoing outbreak has been declared by the World Health Organization (WHO) as a global public health emergency. On March 8, 2020, according to the WHO, 100 countries have reported COVID-19 cases and the number of positive cases passed 100,000 cases worldwide and on March 11, 2020, WHO declared that COVID-19 can be characterized as a pandemic [2].

Based on the findings of one of the first published studies on COVID-19, the common symptoms at the onset of illness included fever, cough, and myalgia, or fatigue, while less common symptoms were sputum production, headache, hemoptysis, and diarrhea [1]. However, the symptoms may be more severe in older people, the immunosuppressed cases, and those with some chronic diseases, including diabetes, cancer, and lung disease [3].

Pregnancy is an immunosuppressive condition; thus, pregnant women are at high risk of developing viral infections, including COVID-19 [4]. Evidence indicates that the coronavirus family is responsible for poor outcomes in pregnant women and their neonates [5-7]. Adverse outcomes in pregnancy may include miscarriage, fetal growth restriction, preterm labor, and maternal mortality [3, 8]. Alfaraj et al. estimated the incidence of neonatal adverse outcomes to be nearly $91 \%$ in maternal infection with the Middle East Respiratory Syndrome Coronavirus (MERS-CoV). The adverse effects included admission to the intensive care unit, prematurity, and neonatal mortality [7].

Regarding the high potential of neonatal adverse outcomes, the evidence on vertical transmission of COVID-19 during pregnancy and delivery is very important. The current evidence regarding the vertical transmission of COVID-19 includes few case reports and some observational studies with small sample sizes and the findings are inconsistent and inconclusive $[9,10]$. Chen et al. evaluated the clinical records of 9 pregnant women with COVID-19 infection in Zhongnan Hospital of Wuhan University, Wuhan, China. Amniotic fluid, cord blood, neonatal throat swab, and breast milk samples from six patients were tested, and all samples tested negative for the COVID-19 [9]. Li et al. published a case report presenting a woman with COVID-19 infection in her $35^{\text {th }}$ week of gestation who delivered an infant via cesarean section. The infant was tested negative for COVID-19. They suggested that vertical transmission is unlikely for this virus [10]. Wang et al. reported a pregnant case of positive COVID-19 infection who gave birth to an infant who was tested negative for severe acute respiratory syndrome coronavirus 2 (SARS-CoV-2) based on Reverse Transcription-Ppolymerase Chain Reaction (RT-PCR) [3]. On the other hand, some studies have debated no vertical transmission of COVID-19 [8, 9, 11-14]. A narrative review study pointed out that due to the lack of adequate data on COVID-19 during pregnancy, both mother and fetus should be followed up extensively [4]. Favre et al. insisted on the current lack of data on the consequences of COVID-19 and recommended extended follow-up for infected pregnant women and their fetuses [8].

However, confirmed evidence on the COVID-19 vertical transmission is very crucial and essential in decisionmaking for the management of pregnant women with COVID-19 infection. Therefore, this systematic review and meta-analysis protocol was designed to assess the possibility of vertical transmission of COVID-19.

\section{Methods}

\section{Study type}

All observational studies, including cross-sectional, cohort, case-control studies, as well as case reports and case series published in peer-reviewed journals until the end of July 2020 will be reviewed. Editorials, commentaries, and letters to editors will be excluded from the review. Articles in any language will be included.

\section{Type of participants}

The inclusion criteria for the studies include:

1. Human studies;

2. Studies assessing adult women over the age of 18 years old;

3. Women should have given birth to live child in the past 3 months;

4. Women should be tested positive for COVID-19.

\section{Type of exposure}

The exposure in the studies will be infection with SARS-CoV-2 documented by positive COVID-19 RTPCR [15]. The sensitivity for RT-PCR in detecting CO- 
VID-19 infection was previously reported to be between $95 \%$ and $100 \%[13,14]$. All subjects with at least two positive RT-PCR results for COVID-19 will be considered as COVID-19-infected cases. This diagnosis implies for both mothers and newborn infants.

The RT-PCR is not performed routinely for all pregnant mothers, and only those who present clinical signs and symptoms that make them suspicious of COVID-19 infection and have positive radiological findings for COVID-19 infection will be tested using RT-PCR.

It should be noted that there is not a consensus on the use and indications for the use of diagnostic tests in the case of COVID-19. Because detection methods are evolving over time, the difference in the sensitivity and usage of the tests is a challenge in conducting a review on studies that have been published since the beginning of the pandemic [10, 14]. Therefore, in this review, we will assess the possibility of vertical transmission of COVID-19 based on RT-PCR as the most used diagnostic test. We acknowledge that the use of RT-PCR alone may lead to bias and reduced the accuracy of the findings, but it was the only test for the assessment of COVID-19.

\section{Primary objective}

To assess the possibility of vertical transmission of SARSCOV-2 through mother to child.

\section{Secondary objective}

1. To assess the association between the time of infection (based on gestational age) and vertical transmission of SARS-CoV-2 to newborn. In case sufficient case reports are present for the assessment, the relationship between gestational age at the time of test positivity and vertical transmission will be analyzed;

2. To assess the association between mode of delivery (normal vaginal delivery or cesarean section) and SARS$\mathrm{CoV}-2$ vertical transmission;

3. To assess the association between breastfeeding and SARS-CoV-2 transmission.

The search method used for the study

The international bibliographic databases, including PubMed, Embase, and Web of Science as well as Google scholar will be searched to identify eligible studies. The search strategy will be designed based on preferred reporting items for systematic reviews and meta-analysis (PRISMA) checklist. A PRISMA-P checklist for this protocol is shown in Additional File 1. The search terms include the following keywords based on medical subject heading (MeSH) terms along with free text searching in combination with Boolean operations (AND and OR) (Table 1).

The primary search will be conducted on PubMed and Embase and the search will be improved based on the search results. The final keywords and search terms will be tailored to other databases.

\section{Searching other resources}

The World Health Organization (WHO) website (www. who.int) will be searched for the keywords. Furthermore, the reference list of the identified studies will also be checked manually for similar studies and these studies will also be included in the review.

\section{Data collection}

\section{Study selection}

The Zotero reference manager (https://www.zotero.org/) will be used to merge the identified studies and remove duplicate publications as well as screen the titles and abstracts. The identified studies will be assessed by two authors (SD And NB) independently based on title and abstracts. The articles that meet the inclusion criteria will be selected for the review. In case of discrepancies, a third reviewer (TFN) will be asked to judge whether to include or exclude the study. In the next step, the full text of the studies will be reviewed by the authors.

\section{Data extraction}

All authors will contribute to data extraction from identified studies. Extracted data will be summarized in a checklist, which is designed by the authors of this review. Based on this checklist, the following data will be extracted:

1. General characteristics of the study (author names, title, publication date, and review date);

2. Type of the study;

3. Sample size;

4. Subjects' characteristics (demographic characteristics, predisposing conditions, gestational age);

5. Outcome measures and analyses (the diagnostic test used and the number of positive samples);

6. Study findings. 
Table 1. The keyword search for the study

\begin{tabular}{|c|c|}
\hline Keyword & Search Terms \\
\hline Coronavirus 2019 & $\begin{array}{l}\text { COVID-19 } \\
\text { COVID-19 } \\
\text { COVID-19 infection } \\
\text { COVID-19 outbreak } \\
\text { COVID-19 pneumonia } \\
\text { SARS-Cov-2 } \\
\text { SARS-Cov-2 } \\
\text { SARS-Cov-2 } \\
\text { SARS-Cov-2 } \\
\text { SARS-Cov-2 } \\
\text { SARS-Cov-2 }\end{array}$ \\
\hline 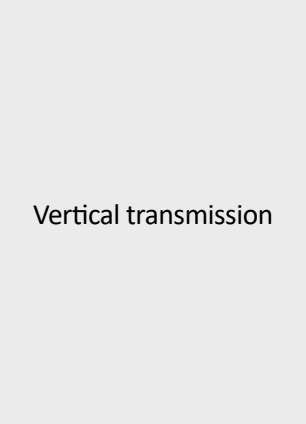 & $\begin{array}{l}\text { Vertical transmission } \\
\text { Vertical transmission rate } \\
\text { Vertical transmission rates } \\
\text { Vertical transmission risk } \\
\text { Vertical transmission risks } \\
\text { Vertical transmission route } \\
\text { Vertical transmission studies } \\
\text { Vertical transmission study } \\
\text { Vertical transmissions } \\
\text { Vertical transovarial transmission } \\
\text { Vertical transplacental transmission }\end{array}$ \\
\hline PubMed search strategy & 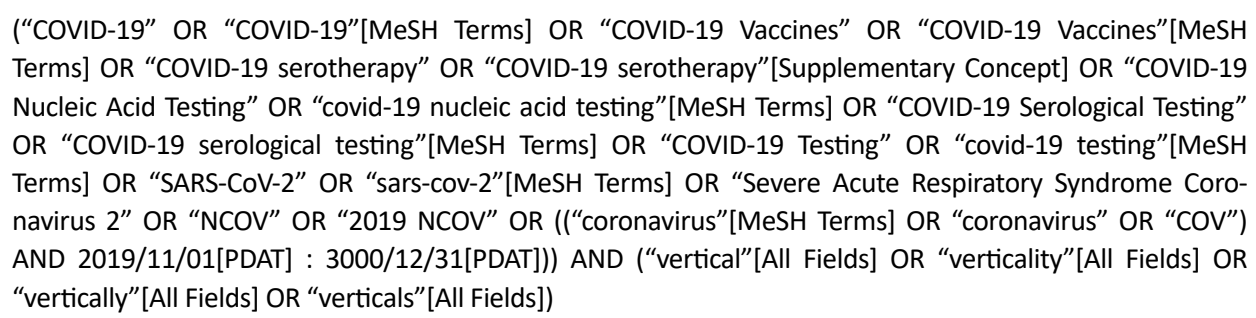 \\
\hline
\end{tabular}

SARS-Cov-2: Severe Acute Respiratory Syndrome Coronavirus-2.

IRA

\section{Quality assessment of the studies}

The quality of the identified studies will be assessed by two authors (SD and NB) independently. As the studies in this review include cross-sectional, cohort, case-control, case reports, and case series studies, the Cochrane Collaboration software and review manager (revMAn) software version 5.0 to assess the risk of bias.

\section{Data analysis}

Heterogeneity among studies will be assessed by DerSimonian \& Laird Q test and $\mathrm{I}^{2}$ statistic, which is the proportion of total variation due to between-studies heterogeneity. To calculate the pooled prevalence estimate of vertical transmission and corresponding 95\% Confidence Intervals (CIs), we will use the Metaprop [15], a STATA-based command developed for binominal data.
To combine risk ratio in cohort studies and odds ratio in case-control studies for assessing the association of gestational age for acquiring the infected in mothers, delivery mode, and breastfeeding with vertical transmission of COVID-19, we will apply a random-effects model in case of heterogeneity among studies. Otherwise, a fixed-effects model will be used. Begg's rank correlation and the Egger weighted regression methods will be used to statistically assess the publication bias. We will also visually assess the publication bias using a funnel plot. P-value $<0.05$ will be considered statistically significant in all tests. Stata version 12 (Stata Corp, College Station, Texas) will be used for all statistical analyses.

\section{Discussion}

This systematic review will assess the possibility of vertical transmission of SARS-CoV-2 from infected mother to child through the transurethral, vaginal, and breast milk routes. The findings of this review will also 
identify the effect of the duration of COVID-19 infection based on gestational age and the possibility of vertical transmission of SARS-CoV-2 to the infant. The findings of this study will help healthcare providers and physicians to identify the risk of vertical transmission in pregnant women infected with COVID-19.

\section{Declaration}

\section{Consent for Publication}

The Research Council of Gonabad University of Medical Sciences approved the proposal of this study and provided written consent for publication of the protocol and the results.

\section{Availability of data and materials}

The search strategy, checklists, and the list of the retrieved articles will be provided online. The full text of the articles will be provided based on the availability of the full text (only open access articles will be provided online).

\section{Ethical Considerations}

\section{Compliance with ethical guidelines}

This study was approved and sponsored by the Research Council of Gonabad University of Medical Sciences (Grant number: A-10-1296-6). Systematic review registration: CRD42020173886.

\section{Funding}

This study was sponsored by the research council of Gonabad University of Medical Sciences (Grant number: A-10-1296-6).

\section{Authors' contributions}

All authors equally contributed to preparing this article.

\section{Conflict of interest}

The authors declared no conflict of interest.

\section{Acknowledgments}

The author would like to thank the Gonabad University of Medical Sciences for financial support.

\section{References}

[1] Huang C, Wang Y, Li X, Ren 1, Zhao J, HU Y, et al. Clinical features of patients infected with 2019 novel coronavirus in Wuhan, China. Lancet. 2020; 395(10223):497-506. [DOI:10.1016/S0140-6736(20)30183-5]

[2] WHO. Rolling updates on coronavirus disease (COVID-19) [Internet] 2020. [Updated 2020 July 31]. Available from: https://www.who.int/emergencies/diseases/novel-coronavirus-2019/events-as-they-happen

[3] Wang X, Zhou Z, Zhang J, Zhu F, Tang Y, Shen X. A case of 2019 Novel Coronavirus in a pregnant woman with preterm delivery. Clin Infect Dis. 2020;10. [DOI:10.1093/cid/ciaa200] [PMID] [PMCID]

[4] Meijer WJ, van Noortwijk AG, Bruinse HW, Wensing AM. Influenza virus infection in pregnancy: A review. Acta Obstet Gynecol Scand. 2015; 94(8):797-819. [DOI:10.1111/ aogs.12680] [PMID]

[5] Maxwell C, McGeer A, Tai KFY, Sermer M. No. 225-Management guidelines for obstetric patients and neonates born to mothers with suspected or probable Severe Acute Respiratory Syndrome (SARS). J Obstet Gynaecol Can. 2017; 39(8):e130-e7. [DOI:10.1016/j.jogc.2017.04.024] [PMID] [PMCID]

[6] Alserehi H, Wali G, Alshukairi A, Alraddadi B. Impact of Middle East Respiratory Syndrome coronavirus (MERS$\mathrm{CoV}$ ) on pregnancy and perinatal outcome. BMC Infect Dis. 2016; 16(1):1-4. [DOI:10.1186/s12879-016-1437-y] [PMID] [PMCID]

[7] Alfaraj SH, Al-Tawfiq JA, Memish ZA. Middle East Respiratory Syndrome Coronavirus (MERS-CoV) infection during pregnancy: Report of two cases \& review of the literature. J Microbiol Immunol Infect 2019; 52(3):501-3. [DOI:10.1016/j jmii.2018.04.005] [PMID] [PMCID]

[8] Favre G, Pomar L, Musso D, Baud D. 2019-nCoV epidemic: What about pregnancies? Lancet. 2020; 395(10224):e40 [DOI:10.1016/S0140-6736(20)30311-1]

[9] Chen H, Guo J, Wang C, LUO F, Yu X, Zhang W, et al. Clinical characteristics and intrauterine vertical transmission potential of COVID-19 infection in nine pregnant women: A retrospective review of medical records. Lancet. 2020; 395(10226):809-15. [DOI:10.1016/S0140-6736(20)30360-3]

[10] Li Y, Zhao R, Zheng S, Chen X, Wang J, Sheng X, et al. Lack of vertical transmission of severe acute respiratory syndrome coronavirus 2, China. Emerg Infect Dis. 2020; 26(6):1335-6. [DOI:10.3201/eid2606.200287] [PMID] [PMCID]

[11] Mardani M, Pourkaveh B. A controversial debate: Vertical transmission of COVID-19 in pregnancy. Arch Clin Infect Dis. 2020; 15(1):e102286. [DOI:10.5812/archcid.102286]

[12] Chu DKW, Pan Y, Cheng SMS, Hui KPY, Krishnan P, Liu $Y$, et al. Molecular diagnosis of a novel Coronavirus (2019$\mathrm{nCoV})$ causing an outbreak of pneumonia. Clin Chem. 2020; 66:549-55. [DOI:10.1093/clinchem/hvaa029] [PMID] [PMCID]

[13] Corman VM, Landt O, Kaiser M, Molenkamp R, Meijer A, Chu DK, et al. Detection of 2019 novel coronavirus (2019-nCoV) by real-time RT-PCR. Euro Surveill. 2020; 25(3):2000045. [DOI:10.2807/1560-7917. ES.2020.25.3.2000045] [PMID] [PMCID] 
[14] Liu YSL, Zhang D, Tang S, Chen H, Chen L, HeX, et al. The epidemiological and clinical characteristics of 2019 novel coronavirus infection in Changsha, China. Lancet. 2020; 1-14. [DOI:10.2139/ssrn.3537093]

[15] Nyaga VN, Arbyn M, Aerts M. Metaprop: A Stata command to perform meta-analysis of binomial data. Arch Public Health. 2014; 72(1):39. [DOI:10.1186/2049-3258-72-39] [PMID] [PMCID] 